\title{
Combined Low-Dose Contrast-Enhanced MR Angiography and Perfusion for Acute Ischemic Stroke at 3T: A More Efficient Stroke Protocol
}

\author{
K. Nael, A. Meshksar, B. Ellingson, M. Pirastehfar, N. Salamon, P. Finn, D.S. Liebeskind, and J.P. Villablanca
}

\begin{abstract}
BACKGROUND AND PURPOSE: There is need to improve image acquisition speed for MR imaging in evaluation of patients with acute ischemic stroke. The purpose of this study was to evaluate the feasibility of a 3T MR stroke protocol that combines low-dose contrastenhanced MRA and dynamic susceptibility contrast perfusion, without additional contrast.
\end{abstract}

METHODS: Thirty patients with acute stroke who underwent 3T MR imaging followed by DSA were retrospectively enrolled. TOF-MRA of the neck and brain and 3D contrast-enhanced MRA of the craniocervical arteries were obtained. A total of $0.1 \mathrm{mmol} / \mathrm{kg}$ of gadolinium was used for both contrast-enhanced MRA $(0.05 \mathrm{mmol} / \mathrm{kg})$ and dynamic susceptibility contrast perfusion $(0.05 \mathrm{mmol} / \mathrm{kg})(\mathrm{referred} \mathrm{to} \mathrm{as}$ half-dose). An age-matched control stroke population underwent TOF-MRA and full-dose $(0.1 \mathrm{mmol} / \mathrm{kg})$ dynamic susceptibility contrast perfusion. The cervicocranial arteries were divided into 25 segments. Degree of arterial stenosis on contrast-enhanced MRA and TOF-MRA was compared with DSA. Time-to-maximum maps ( $>6$ seconds) were evaluated for image quality and hypoperfusion. Quantitative analysis of arterial input function curves, SNR, and maximum $\mathrm{T}^{*}$ effects were compared between half- and full-dose groups.

RESULTS: The intermodality agreements $(k)$ for arterial stenosis were 0.89 for DSA/contrast-enhanced MRA and 0.63 for DSA/TOF-MRA. Detection specificity of $>50 \%$ arterial stenosis was lower for TOF-MRA (89\%) versus contrast-enhanced MRA (97\%) as the result of overestimation of $10 \%$ (39/410) of segments by TOF-MRA. The DWI-perfusion mismatch was identified in both groups with high interobserver agreement $(r=1)$. There was no significant difference between full width at half maximum of the arterial input function curves $(P=$ .14) or the SNR values (0.6) between the half-dose and full-dose groups.

CONCLUSIONS: In patients with acute stroke, combined low-dose contrast-enhanced MRA and dynamic susceptibility contrast perfusion at $3 \mathrm{~T}$ is feasible and results in significant scan time and contrast dose reductions.

ABBREVIATIONS: AIF = arterial input function; CE-MRA = contrast-enhanced MRA; FWHM = full width at half maximum; GRAPPA = generalized autocalibrating partially parallel acquisition; Tmax = time-to-maximum

$\mathbf{S}$ troke is a common and serious disorder, with an annual incidence of approximately $795,000 .^{1}$ It has been estimated that for every minute in which ischemic stroke is left untreated, approximately 1.9 million neurons are lost. ${ }^{2}$ Therefore, both the speed of diagnosis and efficacy of treatment are desirable.

MRA to evaluate the status of both cervical and intracranial arteries and dynamic susceptibility contrast perfusion imaging to

Received May 1, 2013; accepted after revision October 31.

From the Department of Medical Imaging (K.N., A.M.), University of Arizona, Tucson, Arizona; and Department of Radiological Sciences (B.E., M.P., N.S., P.F., J.P.V.) and Department of Neurology, Stroke Center (D.S.L.), University of California, Los Angeles, Los Angeles, California.

Please address correspondence to Kambiz Nael, MD, University of Arizona Medical Center, Department of Medical Imaging, Neuroradiology Section, 1501 North Campbell, PO Box 245067, Tucson, AZ 85724-5067; e-mail: kambiz@radiology. arizona.edu

Evidence-Based Medicine Level 2.

http://dx.doi.org/10.3174/ajnr.A3848 determine the presence of hypoperfused tissue at risk have been used in comprehensive stroke protocols.

Although the concept of applying contrast-enhanced MRA (CE-MRA) in the evaluation of both neck and intracranial arterio-occlusive disease has been explored in the literature, ${ }^{3-5} \mathrm{CE}$ MRA has not been widely adopted into acute stroke protocols for several reasons. First, CE-MRA generally has a lower spatial resolution relative to TOF-MRA because the competing requirements of coverage and acquisition speed require a compromise in CEMRA spatial resolution. A second potential limitation to the incorporation of CE-MRA into clinical stroke protocols is the extra contrast dose required, which is in addition to the intravenous contrast bolus normally used for perfusion imaging. Promising results on low-dose CE-MRA of the supra-aortic arteries have been reported with contrast dose as low as 0.05 $\mathrm{mmol} / \mathrm{kg}$. ${ }^{6,7}$ The effects of contrast dose reduction on DSC perfusion have been evaluated by several investigators, ${ }^{8,9}$ and 
contrast dosages as low as $0.05 \mathrm{mmol} / \mathrm{kg}$ have been used with promising results. ${ }^{10,11}$

The purpose of this study was to prospectively evaluate the feasibility of a combined low-dose CE-MRA and DSC perfusion acute stroke protocol at $3 \mathrm{~T}$ that requires no additional contrast during diagnostic assessment and to compare the results with a routine stroke protocol that employs TOF-MRA. Two hypotheses were tested: 1) low-dose CE-MRA provides comparable or superior diagnostic performance in the detection of both extracranial and intracranial arterio-occlusive disease in comparison with TOF-MRA, with potential for significant reduction in acquisition time; 2) DSC perfusion by use of half of the conventional dose is feasible with comparable image quality.

\section{MATERIALS AND METHODS}

This retrospective study was conducted between August 2011 and April 2013. All examinations were performed in accordance with institutional review board guidelines with an approved study protocol. Inclusion criteria included 1): patients with clinical suspicion of acute ischemic stroke who presented within the first 9 hours from symptom onset; 2) acquisition of 3T MR imaging as the initial imaging test; and 3) DSA within 24 hours after symptom onset. DSA was performed either because of failed response to conventional treatment, including IV tPA $(n=4)$, or detection of a proximal clot and major arterial occlusion requiring further endovascular treatment $(n=26)$. Exclusion criteria included patients with severe contrast allergy, MR-incompatible implants, glomerular filtration rates $<30 \mathrm{~mL} / \mathrm{min} / 1.73 \mathrm{~m}^{2}$, and severe claustrophobia. The baseline NIHSS scores and median time from stroke onset to MR imaging were documented for each patient.

\section{Image Acquisition}

All MR examinations were performed on a 3T (Trio) clinical MR scanner (Siemens, Erlangen, Germany). A combination 12-channel head $(n=8)$ and neck coil $(n=4)$ was used for radiofrequency signal reception. All patients underwent routine MR stroke protocol, which included DWI, FLAIR, gradient refocused echo, brain 3D TOF-MRA and 2D TOF-MRA of the neck, and DSC perfusion imaging.

MRA sequence parameters were brain TOF-MRA: 3D multislab gradient-echo sequence (TR/TE: $25 / 3.8 \mathrm{~ms}$, flip angle: $18^{\circ}, 5$ slabs each with 30 partitions, FOV: $200 \times 200$ mm, matrix: 256 mm, section thickness: $0.7 \mathrm{~mm}$, generalized autocalibrating partially parallel acquisition (GRAPPA) $\times 2$, acquisition time: 5:50 minutes); neck TOF-MRA: 2D gradient-echo sequence (TR/TE: 21/5.9 ms, flip angle: $45^{\circ}, 110$ partitions, section thickness: $2 \mathrm{~mm}$, FOV: $250 \times 180 \mathrm{~mm}$, matrix: $256 \mathrm{~mm}$, GRAPPA $\times 2$, acquisition time: 3:30 minutes).

A high spatial resolution 3D CE-MRA with centric $k$-space ordering was performed just before the perfusion imaging by use of a fast spoiled-gradient refocused echo sequence in the coronal plane: (TR/TE: $3 / 1.2 \mathrm{~ms}$, flip angle: $25^{\circ}$, FOV: $340 \times 280 \mathrm{~mm}$, matrix: $448 \mathrm{~mm}$ ). One hundred twenty sections with a thickness of $0.8 \mathrm{~mm}$ were acquired in the coronal plane, providing arterial coverage from the aortic arch to the cranial vertex. With the use of a GRAPPA algorithm ${ }^{12}$ with an acceleration factor of 4 , a near isotropic sub-millimeter voxel size $3 \mathrm{D}$ dataset $(0.8 \times 0.9 \times 0.8$ $\mathrm{mm}^{3}$ ) was achieved during a 22 -second acquisition. A modified 2-phase contrast injection scheme ${ }^{13}$ was used to perform CEMRA and DSC perfusion imaging, without need for additional contrast. To accomplish this, a total of $0.1 \mathrm{mmol} / \mathrm{kg}$ of gadolinium (MultiHance, Bracco Diagnostics, Princeton, New Jersey) that is normally used routinely for MR perfusion was diluted with normal saline to a total volume of $50 \mathrm{~mL}$. Three $\mathrm{mL}$ of contrast solution was injected and flushed with $20 \mathrm{~mL}$ of saline to determine the transit time from the arm vein to the cervical carotid arteries. A total of $22 \mathrm{~mL}$ of contrast solution was then injected and flushed with $20 \mathrm{~mL}$ of saline for the CE-MRA acquisition. The contrast injection rate for timing run and CE-MRA was $1.5 \mathrm{~mL} / \mathrm{s}$. Subsequently, the remaining $25 \mathrm{~mL}$ of contrast solution was injected and flushed with $20 \mathrm{~mL}$ of saline at $5 \mathrm{~mL} / \mathrm{s}$ for the MR perfusion scan, which was performed last. The DSC perfusion was performed by use of a single-shot, gradient echo-planar sequence (TR/TE: $1920 / 32 \mathrm{~ms}$, flip angle: $90^{\circ}, 25$ partitions, 5-mm section thickness, and an in-plane voxel size of $1.7 \times 1.7 \mathrm{~mm}^{2}$ ). With the use of GRAPPA $\times 2,45$ dynamic frames were obtained over a 90-second acquisition time.

\section{Image Analysis}

MRA. The source data and reconstructed MRA images by use of MIP were used for image analysis. The cervicocranial arteries were divided into 25 segments, including 1,2: bilateral common carotid arteries; 3,4: bilateral cervical ICAs; 5,6: bilateral petrocavernous ICAs; 7,8: bilateral supraclinoid ICAs; 9-12:bilateral anterior cerebral arteries (A1 and A2 segments); 13-16: bilateral MCAs (M1 and M2 segments); 17,18: bilateral cervical vertebral arteries; 19,20: bilateral intradural segments of vertebral arteries; 21 : basilar artery; and 22-25: bilateral posterior cerebral arteries (P1 and P2 segments).

Arterial segments were examined for stenoses independently by 2 neuroradiologists by use of the CE-MRA and TOF-MRA datasets during separate sessions. Datasets were presented in random order to avoid recall bias. Observers were blinded to results of other imaging. The severity of arterial disease was evaluated by use of a 1-4 grading scale: 1 , no stenosis; 2 , mild stenosis with $\leq 50 \%$ luminal narrowing; 3 , significant stenosis with luminal narrowing $>50$ and $<99 \%$; and 4 , occlusion. When 2 or more stenoses were detected in the same vessel segment, the most severe stenosis was used for grading and analysis. Finally, if necessary, discordant reads on both the CE-MRA and TOF-MRA datasets evaluated for arterial stenosis or occlusion were resolved by consensus review and used to determine the diagnostic accuracy of each technique by use of preprocedural DSA as the reference standard.

DSC Perfusion Analysis. DSC perfusion datasets were processed by use of FDA-approved software (Olea Sphere; Olea Medical, La Ciotat, France). DSC analysis consisted of the following steps: 1) truncation of the first 5 time points in the DSC time-series, 2) calculation of prebolus signal intensity on a voxel wise basis, followed by 3 ) conversion of truncated DSC time-series to a concentration-time curve on the basis of the $\mathrm{T} 2{ }^{\star}$ relaxivity of the contrast agent. The change in signal intensity with respect to baseline was 

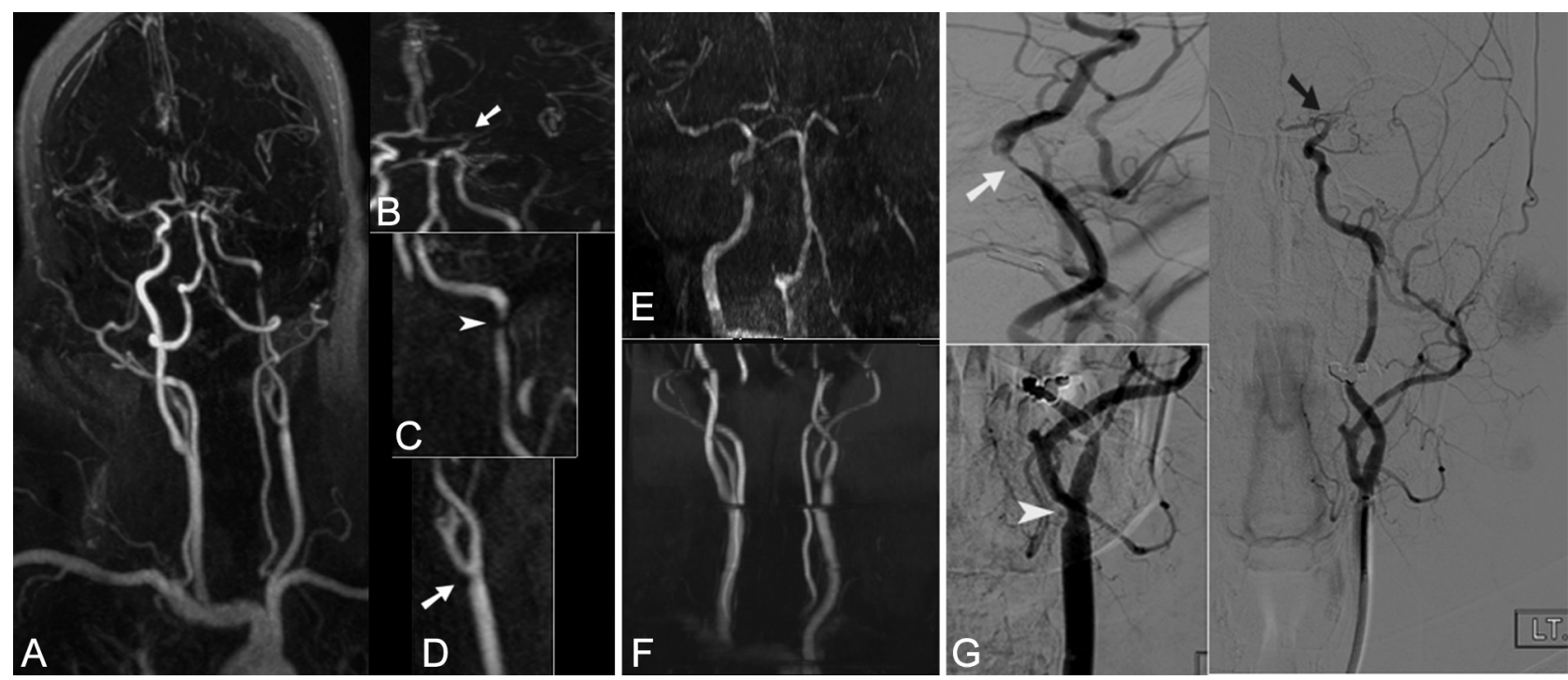

FIG 1. A 55-year-old woman presented with acute onset aphasia; NIHSS score 20. Full-thickness MIP of the entire supra-aortic arteries (A) and focused oblique reformatted (B, $C$, and $D$ ) thin MIP images from CE-MRA, coronal MIP images from TOF-MRA of the brain (E) and neck (F), and focused oblique views from DSA $(G)$ after left common and ICA injections are demonstrated. Both CE-MRA and DSA demonstrate mild stenosis of the left distal common carotid artery (arrow in $D$ and arrowhead in $G$ ), high-grade stenosis of the distal left ICA near the petrous segment (arrow on $C$ and $G$ ), and occlusion of the ICA bifurcation with extension into the left M1 (arrow in $B$ and black arrow in $G$ ). Because of longer acquisition time, both TOF-MRA of the neck (F) and brain (E) are degraded by motion artifact. The mild stenosis at the left common carotid artery is not clearly seen. In addition, because of distal occlusion and sluggish flow, the entire left high cervical, petrocavernous, and supracliniod ICA segments appear to be occluded.

converted to the change in tissue $\mathrm{T} 2{ }^{\star}$ as a result of contrast agent injection, taken with respect to precontrast baseline time points. The arterial input function (AIF) was selected automatically by the perfusion software, and the deconvoluted perfusion parameters were calculated by use of a block-circulant singular value decomposition technique. ${ }^{14}$ Quantitative analysis of the AIF curves was performed to determine the peak and full width at half maximum (FWHM). The infarction volume was automatically calculated by means of the software, by use of ADC threshold $<600 \times 10^{-6} \mathrm{~mm}^{2} / \mathrm{s}$. The time-to-maximum maps, with the use of a threshold of $>6$ seconds, were evaluated by observers independently in each group to determine the presence of hypoperfusion and DWI-perfusion mismatch by use of a 3-scale scoring system: 1 , mismatch present, confident; 2 , mismatch may be present, less confident; 3 , no mismatch present.

The volumes of DWI abnormality, hypoperfused region by use of Tmax $>6$ seconds, and DWI-diffusion mismatch ratios were generated automatically by the software. These values were used as reference to compare the accuracy of subjective mismatch scores (Fig 2).

Quantitative analysis of the SNR values and maximum T2* effect was performed by use of an ROI-based analysis. ROIs approximately $1 \mathrm{~cm}$ in diameter were placed on the normal contralateral postcentral gyrus and corresponding subcortical white matter. The signal intensity of these ROIs was calculated over the entire 45 sequential dynamic perfusion frames, and the mean \pm SD of the signal intensity was noted for each perfusion scan. Maximum $\mathrm{T}^{\star}$ effect, which is the maximum signal intensity drop during the intracerebral passage of an intravenous contrast bolus, was also calculated for each scan. To minimize the nonuniform distribution of noise across the field of view caused by parallel imaging, 2 ROIs were placed in the extracranial space ( 1 at the top and 1 at the bottom of field of view) to determine the background noise. The standard deviation of the mean of these background ROIs in all 45 dynamic frames (90 ROIs) was defined as image noise and was used to calculate the SNR. The baseline SNR was also evaluated by use of the precontrast perfusion images, which were available during the first 4 dynamic frames (initial 8 seconds) before contrast arrival. This was performed to investigate whether the half-dose perfusion scans obtained after CE-MRA were contaminated with recirculating contrast agent and/or had a reduced baseline signal in comparison to the full-dose control perfusion group.

\section{Statistical Analysis}

Statistical analysis was performed by use of MedCalc (Version 12.2.1, MedCalc Software Mariakerke, Belgium). The degree of interobserver and intermodality agreement for estimation of arterial stenosis was determined by kappa $(\kappa)$ coefficients and calculation of $95 \%$ CI. The sensitivity and specificity of CE-MRA and TOF-MRA for stenosis $>50 \%$ was calculated for each observer, by use of preprocedural DSA images as the standard of reference. Spearman-Rank correlation coefficient was used to evaluate the qualitative scores of perfusion maps and interobserver confidence. The SNR values, maximum $\mathrm{T}^{\star}$ effects, and peak and FWHM of the AIF curves were plotted as mean \pm SD, and the differences between the full- and half-dose groups were evaluated by means of a $t$ test. The significance level was defined as $P<.05$ (2-sided).

\section{Cohort Controlled Group}

To perform a comparative analysis for the DSC perfusion parameters, an age-matched control population of 30 patients (12 women, 18 men; age range, 43-90 years; average, 69 years) with 

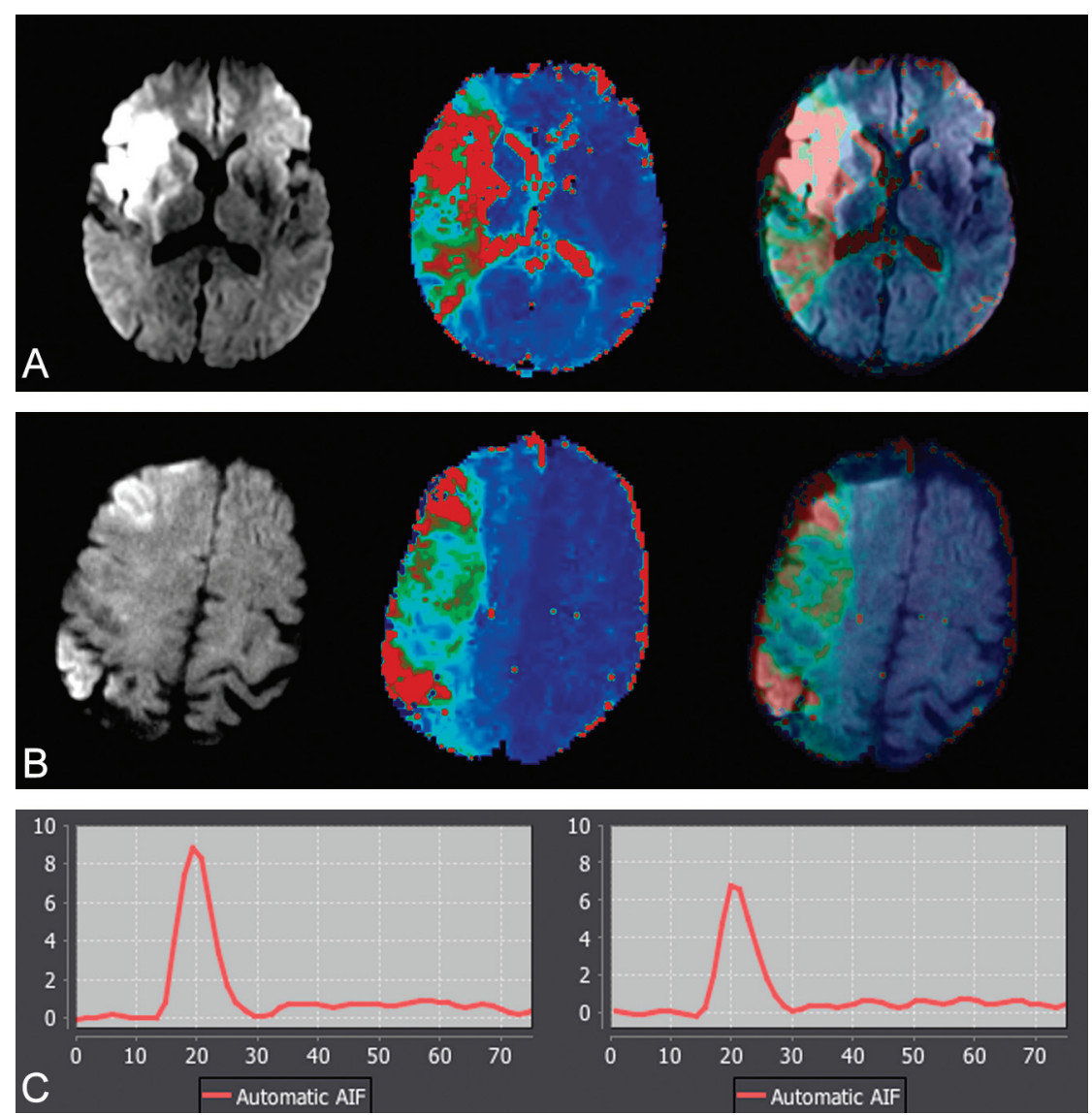

FIG 2. A, 62-year-old man with acute stroke; NIHSS score, 14. MR imaging was performed at 3T, 5 hours after the onset (from cohort group: $0.1 \mathrm{mmol} / \mathrm{kg}$ of gadolinium used for dynamic susceptibility contrast perfusion). B, 58-year-old man with acute stroke; NIHSS score, 16. MR imaging was performed at 3T, 3 hours after the onset (from study group: $0.05 \mathrm{mmol} / \mathrm{kg}$ gadolinium was used for dynamic susceptibility contrast perfusion). Serial DWI, Tmax, and superimposed DWI-Tmax demonstrate diagnostic image quality in both patients. A large perfusiondiffusion mismatch was identified with high confidence in both patients. The corresponding arterial input function curves are shown. There is approximately $24 \%$ lower arterial peak in half dose (6.7) compared with full dose (8.9). There is only small difference between the full width at half maximum of full dose (13) versus half dose (15).

acute stroke who had undergone full-dose contrast $(0.1 \mathrm{mmol} / \mathrm{kg})$ DSC perfusion was selected. The available NIHSS score in the control group ranged from 5-18. In this population, MRA was acquired by use of TOF-MRA only. No CE-MRA was performed before DSC imaging. The studies were performed on an identical $3 \mathrm{~T}$ scanner. The DSC perfusion sequence parameters were equivalent to our study population. A total of 17 patients from the control group had proximal arterial occlusion (internal carotid artery, $n=5$, proximal MCA, $n=12$ ).

\section{RESULTS}

A total of 30 patients ( 18 men; age range, 38 - 84 years; average, 62 years) met our inclusion criteria. Baseline NIHSS scores ranged from 4-24, with a median of 16 . The median time from presentation to MR imaging was 5 hours (range, 1-8 hours).

All studies were completed successfully. In 2 patients (7\%), evaluation of the aortic arch and proximal aspects of supra-aortic arteries on CE-MRA was degraded by motion artifact. In 15 studies (50\%), the image quality of TOF-MRA of the neck was degraded by motion artifact (Fig 1). A total of 52 segmental arterial stenoses were identified by CE-MRA: mild ( $\leq 50 \%): n=14$; high-grade $(>50-$ 99\%): $n=18$; and occlusion: $n=20$. Eighty-two segmental arterial stenoses were identified by TOF-MRA: mild $(\leq 50 \%): n=20$; high-grade $(>50-$ 99\%): $n=22$; and occlusion: $n=40$. The interobserver agreements were $k=0.78$; 95\% CI, 0.65-0.90 for CE-MRA; $k=0.86$; 95\% CI, 0.78-0.92 for TOF-MRA.

Preprocedural DSA images were available for 410 arterial segments, and identified 50 arterial stenoses: mild ( $\leq 50 \%)$ : $n=19$; high-grade (>50\%-99\%): $n=$ 12; and occlusion: $n=19$. Table 1 shows the distribution of arterial stenoses detected by TOF-MRA and CE-MRA in comparison to DSA. Table 2 shows the diagnostic performance and agreement of CE-MRA and TOF-MRA in comparison with DSA as the standard of reference. Compared with DSA, by use of CEMRA, 1 arterial segment with high-grade stenosis was graded as occlusion and 8 arterial segments with mild stenosis were graded high-grade stenoses. This resulted in a sensitivity and specificity of $100 \%$ and $97 \%$, respectively, for the detection of high-grade arterial stenosis (>50\%). With the use of TOF-MRA, 21 arterial segments with varying degrees of stenosis were graded as occluded and 18 arterial segments with no to mild stenosis were graded as high-grade stenosis. This resulted in sensitivity and specificity of $100 \%$ and $89 \%$, respectively, for the detection of high-grade arterial stenosis $(>50 \%)$.

\section{DSC Perfusion}

The mean \pm SD of the volume of infarction on DWI and hypoperfusion with Tmax $>6$ seconds were $16.5 \pm 7.6(\mathrm{~mL}) / 120 \pm 48$ $(\mathrm{mL})$ for the full-dose group and $18 \pm 9(\mathrm{~mL}) / 137 \pm 70(\mathrm{~mL})$ for the half-dose group $(P=.2)$. The mismatch ratios calculated automatically by the software demonstrated DWI-perfusion mismatch in all patients in both groups. DWI-perfusion mismatch was identified in $100 \%$ of the full-dose group and $90 \%$ of the half-dose group by both observers, with high confidence $(r=1)$.

There was no statistically significant difference in the SNR and background noise values between half- and full-dose groups (Table 3). When the baseline SNR of the initial phase of the perfusion study before arrival of contrast was evaluated, there was no significant difference $(P=.6)$ between the mean baseline SNR of the full-dose $(342 \pm 101)$ and half-dose groups $(330 \pm 67)$, indicating an absence of significant recirculation effects. The maximum $\mathrm{T} 2{ }^{*}$ signal drop values were higher in the full-dose group $(P=.01)$. There was statistically significant higher AIF peak in the full-dose group $(P=.002)$. There was however, no statistically significant 
Table 1: Distribution of arterial stenoses detected by TOF-MRA and CE-MRA in comparison with DSA (total arterial segments available for comparison $=410$ )

\begin{tabular}{|c|c|c|c|c|c|c|c|c|c|}
\hline \multirow[b]{3}{*}{ Arterial Segment } & \multicolumn{3}{|c|}{ TOF-MRA } & \multicolumn{3}{|c|}{ CE-MRA } & \multicolumn{3}{|c|}{ DSA } \\
\hline & \multicolumn{3}{|c|}{ Stenosis Severity } & \multicolumn{3}{|c|}{ Stenosis Severity } & \multicolumn{3}{|c|}{ Stenosis Severity } \\
\hline & $\leq 50 \%$ & $>50,<99 \%$ & Occlusion & $\leq 50 \%$ & $>50,<99 \%$ & Occlusion & $\leq 50 \%$ & $>50,<99 \%$ & Occlusion \\
\hline Common carotid artery & 3 & 2 & 3 & 5 & 1 & 0 & 3 & 0 & 0 \\
\hline Cervical ICA & 2 & 1 & 6 & 4 & 4 & 4 & 7 & 5 & 4 \\
\hline Pretrocavernous ICA & 2 & 3 & 4 & 1 & 1 & 1 & 0 & 2 & 1 \\
\hline Supraclinoid ICA & 3 & 4 & 6 & 1 & 5 & 3 & 1 & 1 & 3 \\
\hline MCA & 3 & 6 & 14 & 1 & 2 & 11 & 2 & 1 & 10 \\
\hline Anterior cerebral artery & 2 & 2 & 2 & 0 & 1 & 0 & 2 & 1 & 0 \\
\hline Cervical vertebral artery & 0 & 0 & 1 & 1 & 0 & 1 & 2 & 0 & 1 \\
\hline Intradural vertebral artery & 3 & 0 & 3 & 1 & 0 & 0 & 0 & 1 & 0 \\
\hline Basilar artery & 2 & 1 & 0 & 0 & 0 & 0 & 0 & 0 & 0 \\
\hline Posterior cerebral artery & 0 & 3 & 1 & 0 & 2 & 0 & 2 & 1 & 0 \\
\hline Total & 20 & 22 & 40 & 14 & 18 & 20 & 19 & 12 & 19 \\
\hline
\end{tabular}

Table 2: Diagnostic performance and agreement of CE-MRA and TOF-MRA for arterial stenosis and occlusion in comparison with DSA

\begin{tabular}{|c|c|c|c|c|c|c|c|c|}
\hline \multirow[b]{2}{*}{ DSA } & \multicolumn{2}{|c|}{ No Disease } & \multicolumn{2}{|c|}{ Mild Stenosis ( $\leq 50 \%)$} & \multicolumn{2}{|c|}{ High Grade (>50\%, <99\%) } & \multicolumn{2}{|c|}{ Occlusion } \\
\hline & CE-MRA & TOF-MRA & CE-MRA & TOF-MRA & CE-MRA & TOF-MRA & CE-MRA & TOF-MRA \\
\hline No disease & 353 & 324 & 7 & 17 & 0 & 14 & 0 & 5 \\
\hline Mild stenosis ( $\leq 50 \%$ ) & 5 & 4 & 7 & 3 & 7 & 4 & 0 & 8 \\
\hline High grade (>50\%, <99\%) & 0 & 0 & 0 & 0 & 11 & 4 & 1 & 8 \\
\hline Occlusion & 0 & 0 & 0 & 0 & 0 & 0 & 19 & 19 \\
\hline
\end{tabular}

Note: $-n=410$ arterial segments. DSA served as reference standard.

Intermodality agreement for CE-MRA: $k=0.89 ; 95 \% \mathrm{Cl}, 0.84-0.92$

Intermodality agreement for TOF-MRA, $k=0.63 ; 95 \% \mathrm{Cl}, 0.60-0.72$.

Table 3: Comparative quantitative analysis of DSC perfusion between full dose $(0.1 \mathrm{mmol} / \mathrm{kg})$ and half dose $(0.05 \mathrm{mmol} / \mathrm{kg})$

\begin{tabular}{lrrl}
\hline $\begin{array}{c}\text { Image Parameter } \\
\text { Evaluated }\end{array}$ & Full Dose & Half Dose & t Test \\
\hline SNR & $342 \pm 101$ & $330.3 \pm 67$ & 0.6 \\
Max T2* signal drop & $120 \pm 54$ & $81.6 \pm 48$ & 0.01 \\
Background noise & $2.5 \pm 0.6$ & $2.7 \pm 0.9$ & 0.4 \\
Peak of AIF & $9.1 \pm 2.4$ & $7.2 \pm 2.0$ & 0.002 \\
FWHM of AIF & $16.8 \pm 2.6$ & $18.2 \pm 2.8$ & 0.14 \\
\hline
\end{tabular}

Note:-Data presented as mean \pm standard deviation.

Max indicates maximum.

difference between the FWHM of the AIF curves among the fulldose and half-dose groups $(P=.1)$ (Table 3$)$.

\section{DISCUSSION}

Our results suggest that the described protocol combining lowdose CE-MRA and DSC perfusion imaging is feasible in the evaluation of patients with acute stroke, without the need for additional contrast. With the use of the described low-dose protocol, near isotropic sub-millimeter voxel sizes of neck and brain arteries were obtained with diagnostic accuracy equal or superior to TOF-MRA when compared with DSA. DSC perfusion imaging with reduced contrast dose was feasible at 3T with comparable qualitative results to a full-dose control group. Importantly, the presence of contrast in the circulating blood of the CE-MRA halfdose group did not negatively affect the image quality nor the quantitative analysis of perfusion data when compared with the control full-dose group.

MRA is used not only to detect the presence of intracranial arterio-occlusive disease but also to detect extracranial tandem stenosis to characterize the stroke mechanism. Despite its broad use in stroke protocols, TOF-MRA requires long acquisition times in the order of 8-11 minutes for the neck and brain arteries.
In addition, spin saturation and phase dispersion caused by slow, in-plane, turbulent, or complex flow ${ }^{15,16}$ can result in overestimation of arterial stenosis and occlusion and increase false-positive rates. It is not uncommon to notice the complete absence of flow signal in intracranial branches distal to a sub-occlusive thrombus or clot (Fig 1).

CE-MRA has not been widely incorporated into routine stroke protocols because of its relatively lower spatial resolution for evaluation of intracranial stenosis and need for extra contrast dose, which, until now, has been required in addition to the contrast used for DSC perfusion imaging. Introduction of multi-coil technology with inherent improved SNR and fast imaging techniques such as GRAPPA ${ }^{12}$ have significantly improved the speed and spatial resolution of CE-MRA ${ }^{17,18}$ while also permitting use of very low contrast doses. ${ }^{6,7}$

With the use of the proposed CE-MRA protocol, sub-millimeter voxel sizes of cervicocranial arteries were obtained by use of $0.05 \mathrm{mmol} / \mathrm{kg}$ of gadolinium contrast. The specificity of CE-MRA was higher than TOF-MRA because of more frequent overestimation of arterial stenosis on TOF-MRA, predominantly caused by slow flow and phase dispersion in arterial segments at or distal to a high-grade stenosis or occlusion. Acquisition time required for MR stroke protocol including DWI, FLAIR, gradient refocused echo, and DSC perfusion is approximately 10 minutes with the use CE-MRA as compared with approximately 20 minutes if TOF-MRA is used. Therefore, the use of CE-MRA instead of TOF-MRA can cut total scan time in half and result in a time savings of approximately 10 minutes.

Although the role of DSC perfusion in the acute stroke setting is still being defined, ${ }^{19,20}$ it is being used with increasing frequency in multiple prospective clinical trials and has become an important component of acute MR stroke protocols in large stroke cen- 
ters. The main application of DSC perfusion in patients with acute ischemic stroke is to identify a perfusion-diffusion mismatch. This is performed in most stroke centers with the use of a perfusion time map such as Tmax, as supported by the current literature.

Our study found that by use of a split dose of contrast and after applying contrast for CE-MRA, diagnostic DSC Tmax perfusion images can be obtained with comparable results to a full-dose control group with acceptable interobserver agreement, consistent with reported literature. ${ }^{10,11}$ Furthermore, we showed that despite a lower AIF peak in the half-dose group, the width of AIF was not significantly different between 2 groups $(P=.14)$. As a result, one can assume that the delay time for the occurrence of the peak value (Tmax) would also not differ significantly. This is consistent with the effects of contrast dose reduction on time variables such as MTT and Tmax, as previously noted by Alger et al. ${ }^{9}$ It is important to recognize that the quantitative values of $\mathrm{CBV}$ and $\mathrm{CBF}$ probably varied between the full-dose and halfdose groups.

The influence of dose reduction on tissue relaxivity $\left(\Delta \mathrm{R} 2^{*}\right)$ and deconvoluted values such as $\mathrm{rCBF}$ has been evaluated by several investigators. ${ }^{8-10}$ A systematic evaluation of the relationship between contrast dose reduction and deconvoluted values was beyond the scope of this report. Reducing the contrast dose can result in a decrease in $\Delta \mathrm{R} 2^{*}$ and thus a drop in peak of AIF as shown by us and others, including Alger et al. ${ }^{9}$ This drop in peak AIF amplitude can possibly lead to inaccuracies in quantitative parameters calculated from deconvolution through additional noise contamination.

General consensus suggests an increase in injection length at a fixed rate, or increase in contrast concentration, leads to a higher peak concentration in the brain. We did not compare the quantitative measure of bolus width between the full- and half-dose groups because these were 2 different populations with possibly different hemodynamic characteristics. However, to minimize the effect of contrast bolus length, we used a similar injection rate $(5 \mathrm{~mL} / \mathrm{s})$ and injection volume for both groups by dilution with saline.

One of the other potential disadvantages of the reduced contrast dose is increase in the noise level, which can certainly lead to inaccuracies in AIF and subsequently affects the deconvoluted perfusion parameters. Although not directly evaluated in this report, the image quality and high confidence in defining the perfusion abnormality between 2 groups indicate that noise was not a major limiting factor for the diagnostic interpretation. Importantly, in our study, the quantitative evaluation of SNR between half- and full-dose groups did not reveal a significant difference. In addition, the mean baseline SNR of 2 groups before arrival of contrast bolus was not statistically significant, suggesting that neither recirculation effects nor baseline signal intensity shifts are of concern during analysis of half-dose data when a contrast dose has been administered for CE-MRA before DSC perfusion.

Our study has several limitations. First is a possible selection bias introduced by the inclusion of patients who also underwent DSA. Usually, DSA is considered when there is an intention to treat major proximal arterio-occlusive disease. Therefore, the results and performance of CE-MRA should be interpreted in this context. Second, the 50\% cutoff used for hemodynamic significant arterial stenosis is somewhat arbitrary because there are no firm guidelines to determine the threshold of hemodynamic significance for the intracranial circulation. Third, the full-dose control group for DSC perfusion analysis was different from our patient population, with differences in underlying hemodynamic status, which can potentially complicate direct comparisons of image quality and quantitation. However, the limitation imposed by total contrast dose precludes the use of the same patients as their own control group. By matching the age, sex, and sequence parameters, we attempted to control for these potential confounding factors. Nevertheless, the comparison of image quality scores between 2 different groups should be interpreted with some caution. The choice of a longer TR (1.9 seconds) used in the current study is less ideal for DSC perfusion. This value was chosen to compensate for a possible SNR penalty that may result when reducing contrast dose. ${ }^{21} \mathrm{It}$ is possible that this choice of TR could have resulted in undersampling of the AIF and therefore overestimation of perfusion parameters. Finally, the combination of parallel acquisition (GRAPPA) and contrast dose reduction may accentuate the effect of noise on image quality and DSC quantitative values, though higher SNR at $3 \mathrm{~T}$ can help to mitigate this effect. Larger-scale clinical studies with concurrent use of different contrast dosage in the same individuals would help to validate our results.

\section{CONCLUSIONS}

Combining low-dose CE-MRA and DSC perfusion at $3 \mathrm{~T}$ is feasible, with diagnostic image quality and without the need for additional contrast. The proposed protocol is significantly faster and more accurate than is the routine stroke protocol by use of TOFMRA and may be adopted into routine clinical practice to streamline the treatment of patients with acute stroke. Our study demonstrates the clinical feasibility of performing DSC perfusion by use of a lower dose of contrast with qualitative measures comparable to a conventional dose group.

Disclosures: J. Paul Finn—UNRELATED: Grants/Grants Pending: Siemens Medical Solutions, Comments: Research grant only indirectly related to the work in this project; Payment for Lectures (including service on speakers bureaus): Honorarium for lecture from Siemens Medical Solutions. David Liebeskind-UNRELATED: Consultancy: Covidien, ${ }^{\star}$ Stryker*; Grants/Grants Pending: $\mathrm{NIH},{ }^{*}$ Comments: Research grants (*money paid to institution).

\section{REFERENCES}

1. Lloyd-Jones D, Adams RJ, Brown TM, et al. Heart disease and stroke statistics-2010 update: a report from the American Heart Association. Circulation 2010;121:e46-e215

2. Saver JL. Time is brain-quantified. Stroke 2006;37:263-66

3. Sohn $\mathrm{CH}$, Sevick RJ, Frayne R. Contrast-enhanced MR angiography of the intracranial circulation. Magn Reson Imaging Clin $\mathrm{N} \mathrm{Am}$ 2003;11:599-614

4. Bernstein MA, Huston J 3rd, Lin C, et al. High-resolution intracranial and cervical MRA at 3.0T: technical considerations and initial experience. Magn Reson Medicine 2001;46:955-62

5. Takano K, Utsunomiya H, Ono H, et al. Dynamic contrast-enhanced subtraction MR angiography in intracranial vascular abnormalities. Eur Radiol 1999;9:1909-12

6. Tomasian A, Salamon N, Lohan DG, et al. Supraaortic arteries: contrast material dose reduction at 3.0-T high-spatial-resolution MR angiography-feasibility study. Radiology 2008;249:980-90 
7. Nael K, Moriarty JM, Finn JP. Low dose CE-MRA. Eur J Radiol 2011;80:2-8

8. Heiland S, Reith W, Forsting M, et al. How do concentration and dosage of the contrast agent affect the signal change in perfusionweighted magnetic resonance imaging? A computer simulation. Magn Reson Imaging 2001;19:813-20

9. Alger JR, Schaewe TJ, Lai TC, et al. Contrast agent dose effects in cerebral dynamic susceptibility contrast magnetic resonance perfusion imaging. J Magn Reson Imaging 2009;29:52-64

10. Manka C, Traber F, Gieseke J, et al. Three-dimensional dynamic susceptibility-weighted perfusion MR imaging at 3.0 T: feasibility and contrast agent dose. Radiology 2005;234:869-77

11. Alger JR, Schaewe TJ, Liebeskind DS, et al. On the feasibility of reduced dose dynamic susceptibility contrast perfusion MRI for stroke. Paper presented at International Society of Magnetic Resonance Medicine, Montréal, Québec, Canada. May 7-13, 2011

12. Griswold MA, Jakob PM, Heidemann RM, et al. Generalized autocalibrating partially parallel acquisitions (GRAPPA). Magn Reson Med 2002;47:1202-10

13. Habibi R, Krishnam MS, Lohan DG, et al. High-spatial-resolution lower extremity MR angiography at 3.0 T: contrast agent dose comparison study. Radiology 2008;248:680-92

14. Wu O, Ostergaard $\mathrm{L}$, Weisskoff RM, et al. Tracer arrival timing-insensitive technique for estimating flow in MR perfusion-weighted imaging using singular value decomposition with a block-circulant deconvolution matrix. Magn Reson Med 2003;50:164-74
15. Lin W, Tkach JA, Haacke EM, et al. Intracranial MR angiography: application of magnetization transfer contrast and fat saturation to short gradient-echo, velocity-compensated sequences. Radiology 1993;186:753-61

16. Isoda $\mathrm{H}$, Takehara $\mathrm{Y}$, Isogai $\mathrm{S}$, et al. MRA of intracranial aneurysm models: a comparison of contrast-enhanced three-dimensional MRA with time-of-flight MRA. J Comput Assist Tomogr 2000;24: 308-15

17. Nael K, Villablanca JP, Pope WB, et al. Supraaortic arteries: contrastenhanced MR angiography at 3.0 T-highly accelerated parallel acquisition for improved spatial resolution over an extended field of view. Radiology 2007;242:600-09

18. Phan T, Huston J 3rd, Bernstein MA, et al. Contrast-enhanced magnetic resonance angiography of the cervical vessels: experience with 422 patients. Stroke 2001;32:2282-86

19. Butcher KS, Parsons M, MacGregor L, et al. Refining the perfusiondiffusion mismatch hypothesis. Stroke 2005;36:1153-59

20. Sobesky J, Zaro Weber O, Lehnhardt FG, et al Does the mismatch match the penumbra? Magnetic resonance imaging and positron emission tomography in early ischemic stroke. Stroke 2005;36: 980-85

21. Carpenter TK, Armitage PA, Bastin ME, et al. DSC perfusion MRI: quantification and reduction of systematic errors arising in areas of reduced cerebral blood flow. Magn Reson Med 2006;55: 1342-49 